\title{
Lineamientos para propuestas metodológicas inclusivas en Matemáticas para estudiantes ciegos
}

\author{
Karen María Ramírez Zuñiga ${ }^{1}$, Estibaliz Rojas Quesada ${ }^{2}$ \& Ronald Sequeira Salazar ${ }^{3}$ \\ 1. Licenciada en Enseñanza de la Matemática en la Universidad de Costa Rica. Tutora del Programa de Enseñanza de \\ la Matemática de la Escuela de Ciencias Exactas y Naturales de la UNED. Correo electrónico: kramirezz@uned.ac.cr. \\ Teléfono: 8864-1355.
}

2. Licenciada en Enseñanza de la Matemática en la Universidad de Costa Rica. Encargada de la cátedra Matemáticas Básicas del Programa de Enseñanza de la Matemática de la Escuela de Ciencias Exactas y Naturales de la UNED. Correo electrónico: erojas@uned.ac.cr.Teléfono: 2202-1845.

3. Dr. en Educación. Encargado del Programa Bachillerato y Licenciatura Enseñanza de la Matemática. Correo electrónico: rsequeira@uned.ac.cr. Teléfono: 2202-1843.

Recibido: 14 de noviembre, 2016

\section{RESUMEN}

Este artículo es el producto de la primera parte del proyecto de investigación "Mediación pedagógica en geometría para la atención de personas ciegas o con baja visión, en sétimo año de la educación costarricense", que se lleva a cabo en el Programa de Bachillerato y Licenciatura Enseñanza de la Matemática y que tiene por objetivo establecer lineamientos generales, que guíen al docente en el diseño de propuestas metodológicas para la mediación pedagógica en Matemáticas. Se consultaron los programas de estudio vigentes de matemática del Ministerio de Educación Pública (MEP), literatura especializada tanto a nivel nacional como internacional y la sistematización de la experiencia obtenida en el Taller Estrategias Inclusivas para el Desarrollo de las Matemáticas en Secundaria, dirigido a profesores de Matemática de San Ramón y San José durante los años 2012 y 2013 en coordinación con la Cátedras de Educación Especial e Informática Educativa de la Escuela de Ciencias de la Educación y la Cátedra de Geometría de la Escuela de Ciencias Exactas y Naturales de la UNED.

Palabras clave: Atención a la diversidad, diseño universal, estudiante ciego, estudiante con baja visión.

\section{SUMMARY}

Guidelines for the inclusive methodological proposals in Mathematic for blind students.

This article is the product of the first part of the research project "Pedagogical mediation in geometry to the attention of blind or low vision, in seventh grade of Costa Rican education" it takes place in the Undergraduate Program Teaching Mathematics and aims establish general guidelines to guide teachers in designing methodological
Aceptado: 01 de diciembre, 2016

proposals for pedagogical mediation in mathematics. For the literature review new curriculum of mathematics at the level of seventh grade the Ministry of Public Education (MEP), specialized literature both nationally and internationally and systematization of the experience gained in Workshop literature consulted Strategies Inclusive Development of mathematics in secondary schools, aimed at teachers of mathematics high school of San Ramon and San Jose during the years 2012 and 2013 in coordination with the Department of Special Education and Educational Informatics at the School of Education Sciences and the Department of Geometry School Natural Sciences of the UNED.

Key words: Attention to diversity, universal design, blind student, student with low vision.

\section{Introducción}

Basado en experiencias que ha tenido la Universidad Estatal a Distancia de Costa Rica (UNED), específicamente en el Programa de Bachillerato y Licenciatura Enseñanza de la Matemática durante el desarrollo de proyectos de extensión en todo el país, se han observado algunas falencias en la mediación pedagógica de los contenidos matemáticos en estudiantes con necesidades educativas especiales (NEE).

Son muchas las NEE que le corresponde atender al profesor de Matemática en las aulas de las instituciones educativas del país, especialmente cuando debe realizar adecuaciones significativas que requieren una mediación 
distinta la cual involucra una diversidad de recursos para que los estudiantes logren un aprendizaje significativo.

El proyecto "Mediación pedagógica en geometría para la atención de personas ciegas o con baja visión, en sétimo año de la educación costarricense", en su primera etapa se enfocó en una investigación bibliográfica de lo escrito en los últimos años sobre la atención a la diversidad, la exclusión social y educativa en el contexto internacional, la discapacidad visual a nivel internacional y en Costa Rica, así como criterios para el diseño de estrategias metodológicas que atienden a la diversidad.

\section{Metodología}

Se utilizaron varias fuentes documentales de las bibliotecas de las universidades públicas e instituciones especializados en la temática como el Centro Nacional de Recursos para la Educación
Inclusiva (CENAREC), el Centro Nacional de Educación Especial Fernando Centeno Güell y la Cátedra de Educación Especial de la Escuela de Ciencias de la Educación de la UNED. También se consultaron otros sitios de interés en la web como blogs y bases de datos, los programas de estudio vigentes de Matemática del Ministerio de Educación Pública (MEP) y en algunos casos se consideró la opinión de especialistas y de profesores con alguna experiencia con estudiantes NEE que participaron en el Taller Estrategias Inclusivas para el Desarrollo de las Matemáticas en Secundaria, dirigido a profesores de Matemática de San Ramón y San José durante los años 2012 y 2013.

La búsqueda se dividió en dos momentos, en el primero se hizo una revisión general de la literatura existente y para ello se confeccionó una ficha resumen. En el cuadro 1 se muestra un ejemplo de esta ficha.

CUADRO 1

Ficha resumen

\begin{tabular}{|l|l|}
\hline Título: Enseñar Matemáticas a alumnos con necesidades educativas especiales. & Signatura: 372.7 \\
M385e2
\end{tabular}


En el segundo momento, se hizo una revisión minuciosa de cada libro consultado, así como de artículos publicados en la web y otros sitios de interés y se sistematizó la información relevante como insumo para el marco teórico de este proyecto. También se consideró el informe final del Taller realizado con los docentes de secundaria en San Ramón y San José durante los años 2012 y 2013.

\section{Desarrollo}

\section{Las NEE en el contexto internacional}

Antes de señalar cuáles son los lineamientos base para la construcción de propuestas metodológicas, primero se debe considerar los fundamentos teóricos que respaldan aspectos como la discapacidad visual, atención a la diversidad y diseño universal.

\section{La exclusión social y educativa en el contexto internacional}

En muchos de los países del mundo, especialmente en África y América Latina, la exclusión social y educativa son fenómenos que han ido creciendo a lo largo de los años. Estas regiones se caracterizan por tener sociedades altamente segmentadas donde el grueso de la población no tiene las mismas condiciones de acceso a las Tecnologías de la Información y la Comunicación (TIC's) ni a la sociedad del conocimiento, esto según la UNESCO citado por Blanco (2009).

Históricamente, los sistemas educativos fueron concebidos como medios de movilidad social e integración de las sociedades a los sistemas de producción para una distribución equitativa de la riqueza, sin embargo, para estas zonas del mundo poco favorecidas, esta visión de lo que debería ser la educación para el desarrollo social, económico y cultural no se ha manifestado de esta forma.

Por el contrario, la educación es el ente social que más ha contribuido a segmentar la sociedad entre ricos y pobres, pues la brecha que existe en la educación pública y privada en estos países está muy marcada, por ejemplo, los jóvenes de las barriadas pobres y marginales de las grandes urbes así como de las zonas rurales alejadas de los centros poblacionales con mayor desarrollo, tienen menor acceso a los servicios educativos y mayores índices de repitencia y abandono del sistema, lo mismo sucede con los inmigrantes que en muchas ocasiones no tienen la oportunidad de aprender en su misma lengua.

Sin embargo, existen otros factores que contribuyen a que el fenómeno de la exclusión social y educativa sea un problema de difícil solución para los organismos internacionales como la ONU, UNESCO, Banco Mundial, entre otros; por ejemplo, la transformación del núcleo familiar y el deterioro en las redes de integración social, han contribuido a que los índices de abandono del sistema educativo se hayan elevado, pues la escuela asume que el peso de la educación de los jóvenes compete a la familia y que no solo debe garantizar las condiciones económicas para que sus hijos asistan diariamente a clases, sino que también tienen que prepararlos desde su infancia para que puedan aprender.

De igual manera, el trabajo realizado por la población menor de edad y la desnutrición, son tan solo otros de los factores que impiden que un alto porcentaje de los niños y jóvenes, principalmente en muchos de los países de América Latina y África, no puedan concluir la educación obligatoria establecida por cada Estado.

Según el Informe de Políticas de Atención a la Diversidad Cultural de la UNESCO y citado por Hirmas (2008):

...La diversidad cultural, en lugar de ser considerada como patrimonio común de la humanidad y oportunidad de crecimiento, se convierte en amenaza, y es utilizada como excusa para la intolerancia y la discriminación. (UNESCO, 2005a)

Por otro lado, en todos los escritos sobre la Declaración Universal de los Derechos Humanos, el principio a la no discriminación está presente y prohíbe todo tipo de discriminación y establece en su artículo uno que "Todos 
los seres humanos nacen libres e iguales en dignidad y derechos". (UN, 2015).

En esta declaración no se hace una alusión específica al tema de la discapacidad, ya que se asume que las personas con discapacidad no requieren de mención especial, en tanto están comprendidas en todos y cada uno de los derechos allí enunciados. (Meléndez, 2010).

De igual manera, la Convención de los Derechos del Niño en la Asamblea General de las Naciones Unidas celebrada el 20 de noviembre de 1989, establece que los Estados tienen que reconocer que el niño mental o físicamente impedido deberá disfrutar de una vida plena y decente, con una participación activa en la comunidad. (Meléndez, 2010).

En Costa Rica, la educación es un derecho básico y de carácter obligatorio, según se establece en su Constitución Política, por lo tanto, todos los individuos deberán tener las mismas oportunidades en el sistema educativo, y el gobierno y la sociedad tienen que asegurar una educación inclusiva de calidad que responda a todos los estudiantes, incluyendo a aquellos que presentan NEE derivadas de una discapacidad física, intelectual, sensorial o de otras condiciones como problemas de aprendizaje, población inmigrante o estudiantes talentosos, entre otros.

Desde esta perspectiva, la inclusión educativa deberá evidenciarse en la mediación pedagógica para que tantos los niños con discapacidad o sin ella puedan aprender juntos ya sea durante todo su proceso de formación o en alguna parte de ella, así entonces, la educación inclusiva tiene que reconocer las diferencias individuales y dar respuesta a esas diferencias en el ámbito educativo.

\section{La discapacidad visual a nivel internacional}

La discapacidad siempre ha estado presente en la historia de la raza humana, por ejemplo, en la antigua Grecia, las personas con discapacidad eran lanzadas desde el Monte Taigeto, pues los griegos le rendían culto a la belleza y a la perfección física, e inclusive existen registros sobre estudios de algunas diferencias físicas y mentales que realizaron los filósofos Diógenes, Hipócrates y Galeno quienes estudiaron la epilepsia y la demencia, entre otras (Cátedra Iberoamericana, 2015).

Es con la aparición del cristianismo que se empiezan a crear instituciones para la atención de la persona con discapacidad y no es hasta el siglo XVIII específicamente en 1784 cuando Valentín Haüy funda en varios países europeos algunas instituciones para la atención de este tipo de población.

En 1830 en los Estados Unidos se inicia un movimiento educativo para la atención de las personas con algún tipo de discapacidad culminando con la creación de la Perkins School for the Blind (Escuela para Ciegos) y The Institute for the Blind (Instituto para Ciegos) en la ciudad de New York en el año de 1832.

En 1852, Louis Braille, crea el sistema de escritura Braille y en 1900 en la ciudad de Chicago se integra por primera vez un estudiante ciego en el sistema educativo convencional, lo que originó que otras ciudades de los Estados Unidos iniciaran sus propios programas educativos para este tipo de estudiantes. En 1921, el Instituto Perkins en colaboración con la Universidad de Harvard, inicia un programa para la formación de docentes en Educación Especial con el objetivo de atender a aquellos estudiantes con algún tipo de discapacidad.

En materia de normativa, en el siglo XX se desarrollan una serie de acciones que se plasman en declaraciones y acuerdos internacionales como la Declaración Universal de los Derechos Humanos de 1948, que es el referente más importante y tiene como propósito brindarle a los países miembros un instrumento que sirva para la promoción del conocimiento de los derechos humanos para todos los ciudadanos del mundo, en tanto asume que las personas con discapacidad están comprendidas en todos los derechos allí enunciados (CENAREC, 2012, p. 15).

Por otro lado, la Asamblea General de la ONU define el Programa de Acción Mundial para las Personas con Discapacidad y en 1989 establece la Convención de los Derechos del Niño. 


\section{La discapacidad visual en Costa Rica}

La atención educativa de estudiantes ciegos o con baja visión es una necesidad que, a nivel nacional, el MEP ha enmarcado dentro de sus prioridades, como una manera de garantizar el acceso y la participación de cada individuo de manera igualitaria. En esta investigación se considera completamente ciego (no vidente) al individuo que presenta una ausencia total de la visión, y baja visión al que todavía conserva un resto visual que le facilita el desplazarse y tener una aprehensión del mundo exterior.

Una de las instituciones pioneras en la atención de estudiantes ciegos en Costa Rica es el Centro Nacional de Educación Especial Fernando Centeno Güell; el cual se crea el 23 de julio de 1940, y posteriormente el 15 de marzo de 1944 mediante la Ley Constitutiva No 61 se declara de interés público la educación de niños con retardo mental, o de trastornos auditivos, de vista o del sistema vocal o de cualquier otro impedimento físico análogo que les impida proseguir con éxito las enseñanzas de la escuela común. (CENAREC, 2012, p. 4)

A partir de ahí, la educación especial en Costa Rica toma otro giro y se empieza a gestar acciones para brindar a todas las personas una educación adecuada a las necesidades educativas que se tienen en la población estudiantil.

El 30 de octubre de 1957 con la Ley 2171, se crea el Patronato Nacional de Ciegos durante la administración de José Figueres Ferrer y cuya creación obedeció a la solicitud de un grupo de personas ciegas que consideraban que era necesaria la unión para lograr objetivos comunes como servicios de bibliotecas con libros en Braille y recursos tecnológicos, becas, el desarrollo de actividades educativas y culturales, entre otras.

En 1977 abre sus puertas la escuela Manuel Ortuño en Desamparados como "una experiencia innovadora bajo la modalidad de aula recurso; a saber, un servicio educativo para atender estudiantes con ceguera y baja visión ubicados(as) en grupos regulares de esa institución" (CENAREC, 2012. p. 7). En 1982, el MEP creó la Asesoría Nacional de Deficiencia Visual, que trabaja en colaboración estrecha con las Asesorías Regionales de Educación Especial del territorio nacional." (CENAREC, 2005. p. 12)

Para el 3 de diciembre de 1985, se crea el "Instituto de Rehabilitación y Formación Helen Keller, como una dependencia de mínima desconcentración del MEP, y cuya responsabilidad es atender las necesidades educativas de la población joven y adulta con ceguera absoluta y baja del país". (CENAREC, 2012. p. 9). El Instituto Helen Keller aún sigue con sus funciones y se cataloga como uno de los centros educativos pioneros en la atención de estudiantes con ceguera o baja visión.

En el año 2000 el Consejo Superior de Educación de Costa Rica, aprueba una serie de disposiciones relacionadas con las ofertas educativas de la educación especial, fundamentadas en la Ley 7600 de Igualdad de Oportunidades para las Personas con Discapacidad, y su reglamento tiene que ver con horarios, programas, opciones de matrícula y otros elementos, en concordancia con los nuevos enfoques de Educación inclusiva. Se aprueban de esta manera, los planes de estudio para aulas integradas y centros de educación especial, así como los planes de estudio para la atención a personas adultas con discapacidad.

En el marco legal, la Ley 7600 en su artículo 22 establece como obligaciones del MEP el suministrar asesoramiento, recursos y la capacitación que se requiera; con el fin de que los alumnos con discapacidad visual, cuenten con una educación adecuada y pertinente. (Costa Rica. Leyes, decretos. 2004, p.13).

Otra institución de especial interés para la educación inclusiva en Costa Rica, es el CENAREC, creado el 14 de diciembre del 2007 mediante la Ley 34206 y que cuenta con el Centro de Producción de Materiales Educativos en Sistema Braille, Relieve y Sonoro (CEBRA), el cual ofrece a la población con discapacidad visual (ceguera y baja visión) diferentes materiales educativos en sistema Braille, en audio, en relieve o en ampliación, según la necesidad del usuario, para mejorar su calidad de vida.

iAdemás, en el año 2008, la Asamblea Legislativa aprueba la Ley 8661, Convención de los Derechos de las Personas con Discapacidad 
y su Protocolo, con la cual el estado costarricense ratifica su disposición a colaborar con lo estipulado en dicha Convención, iniciativa de la ONU.

Finalmente, a pesar de todo lo que se ha escrito en la normativa costarricense con respecto a la atención a la diversidad, lo cierto del caso, es que en el país todavía se requiere de muchos recursos económicos e infraestructura que responda a las diferencias individuales y a las necesidades educativas de este tipo de estudiantes, más aún, el MEP tiene que promover la formulación de programas que atiendan las necesidades educativas especiales, y en los centros educativos deberá hacer las adaptaciones físicas, recurso humano y sugerencias de métodos y técnicas necesarias (adecuaciones curriculares, recursos didácticos, evaluación,... ) para que esto sea una realidad.

En el Vigésimo Informe del Estado de la Nación se señala que un porcentaje muy elevado de personas con discapacidad en edad de trabajar se encuentra fuera de la fuerza laboral. (XX Informe Estado de la Nación, 2014, p.109)

\section{Antecedentes}

Desde el año 2012, la Cátedra de Geometría del Programa de Enseñanza de la Matemática de la Escuela de Ciencias Exactas y Naturales, así como las Cátedras de Educación Especial e Informativa Educativa de la Escuela de Ciencias de la Educación de la Universidad Estatal a Distancia (UNED) han venido realizando esfuerzos importantes para promover la atención a la diversidad y brindar espacios de apoyo para los docentes en ejercicio en esta temática.

Como parte de estas iniciativas se desarrolló el taller titulado "Estrategias inclusivas para el desarrollo de las Matemáticas en secundaria" coordinado por la M.Sc. Ana Patricia Vázquez Chaves de la Cátedra de Educación Especial de la Escuela de Ciencias de la Educación de la UNED, y dirigido a los profesores de Matemática de secundaria pertenecientes a la Dirección Regional Educativa de Occidente en la región de San Ramón durante los meses de setiembre y octubre del 2012 en cinco sesiones de trabajo, todo esto con el propósito de divulgar el Modelo Social de la discapacidad y el Enfoque de Derechos Humanos de la educación en este tipo de población, en el marco específico de la enseñanza de las Matemáticas así como también la incorporación de las TIC disponibles para la enseñanza y aprendizaje de esta asignatura, según Vásquez, (2012).

Posteriormente en el año 2013 se repite la experiencia con un grupo de profesores de Matemática de secundaria de la Dirección Regional Educativa de San José Norte durante los meses de agosto y setiembre. Entre algunos de los tópicos desarrollados en ambos talleres según Vásquez, (2012), se citan:

- Relaciones entre lenguaje y actitudes hacia la diversidad.

- Entornos sin barreras, ¿utopía o esperanza?

- Estrategias inclusivas: el rol del docente.

- Metodologías participativas e inclusivas.

- La diversidad como oportunidad de aprendizaje colectivo.

- La tecnología como herramienta para potenciar la atención a la diversidad desde un enfoque pedagógico.

En las Figuras 1 y 2 se muestra un ejercicio sobre barreras del entorno que realizaron los participantes en estos talleres. Como resultado de estas experiencias, es que el Programa de Enseñanza de la Matemática de la UNED decide presentar en el 2014 el proyecto de investigación titulado "Mediación pedagógica en geometría para la atención de personas ciegas o con baja visión en sétimo año de la educación costarricense" el cual está ubicado en la línea de investigación de la UNED “Aportes para la resolución de problemas que afectan a los grupos de personas en condiciones de vulnerabilidad en Costa Rica" y en la línea de investigación del Programa de Enseñanza de la Matemática "La formación de profesores y el currículo en la educación matemática" y cuyo propósito en esta primera etapa del proyecto es establecer lineamientos generales que sirvan de base para la construcción 


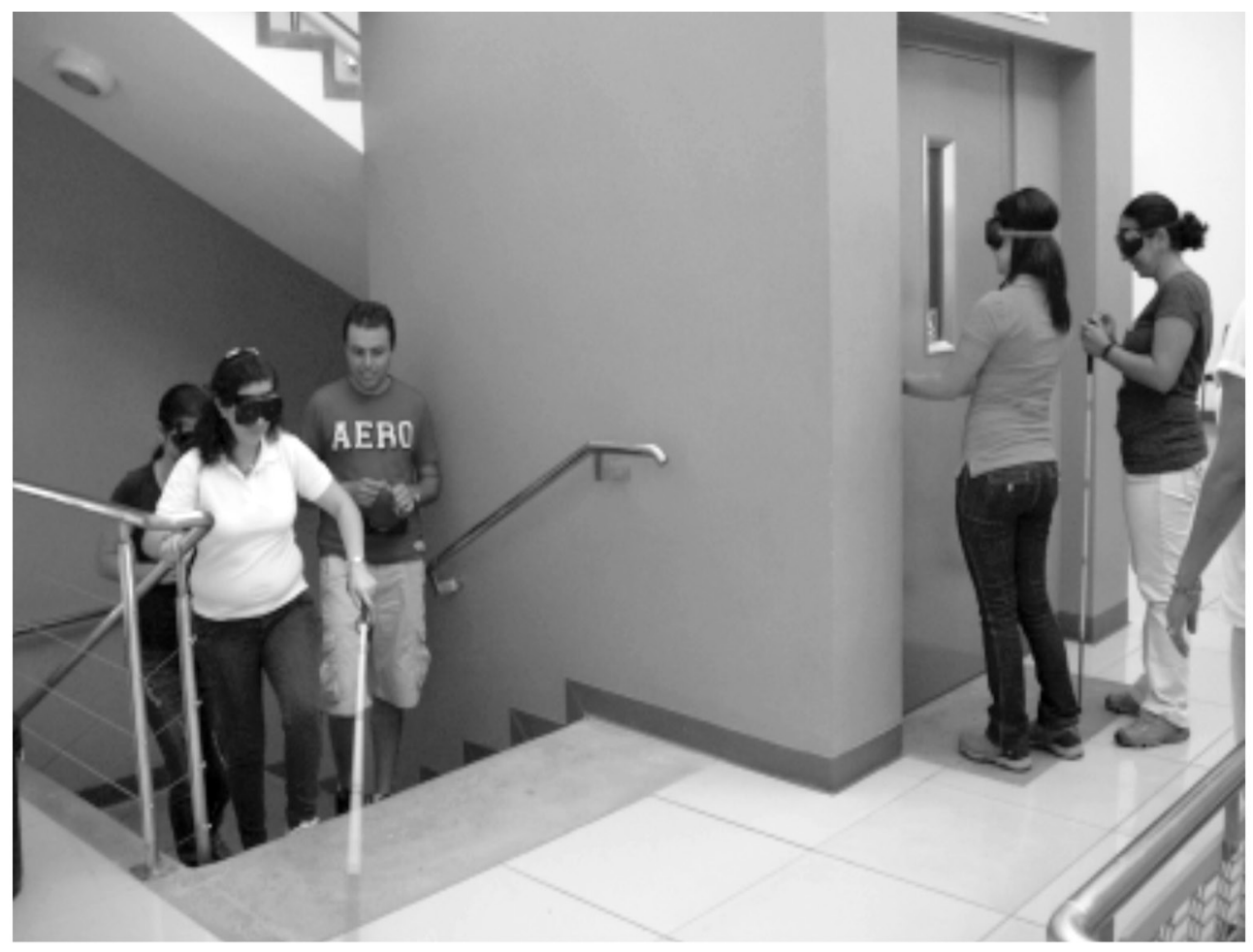

Figura 1. Barreras del entorno.

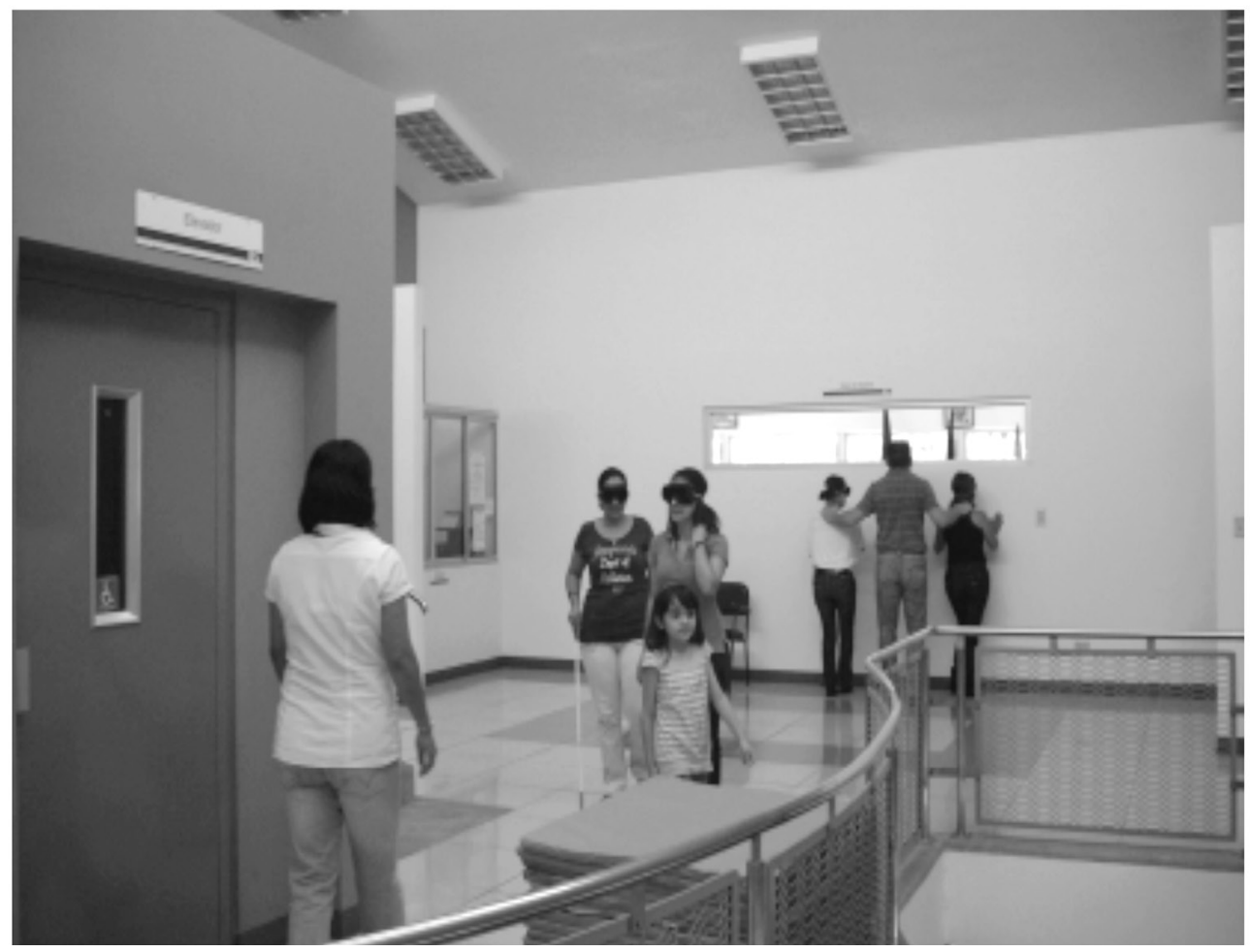

Figura 2. Barreras del entorno. 
de propuestas metodológicas inclusivas en geometría en sétimo año.

\section{Lineamientos generales}

Según la Fundación Centros de Aprendizaje (2010) para elaborar propuestas de inclusión, se debe tomar en cuenta factores como:

- Asesoría a docentes en el conocimiento y manejo de las variaciones en los ritmos y la capacidad de aprendizaje de los estudiantes.

- Contexto sociocultural del espacio intervenido.

- Participación activa de los padres de familia y de la comunidad.

- Trabajo con docentes en red, por área y entre áreas de estudio.

- Creación de grupos de trabajo heterogéneos de estudiantes.

- Seguimiento personalizado al proceso dentro del aula.

Específicamente para construir una propuesta metodológica inclusiva para estudiantes ciegos o de baja visión se recomienda tomar en cuenta los siguientes lineamientos:

\section{Diagnóstico}

Es imprescindible antes de construir una propuesta metodológica conocer el tipo de estudiante al que va dirigida, sus capacidades intelectuales (cómo ha trabajado hasta el momento con otros docentes), su familia: el tipo de apoyo brindado por ésta, además de su nivel socioeconómico y cultural. (Nivel cognitivo, habilidades motoras, el espacio físico (aula). Se recomienda:

1. Valorar las capacidades intelectuales, habilidades, condiciones familiares, socioeconómicas y culturales del estudiante ciego o de baja visión.

2. Recopilar la información mediante una entrevista grabada para cuando se necesite acceder a ella, además de la observación constante del estudiante y la revisión de trabajos que este haya desarrollado.

3. Crear una guía para la entrevista y la observación del estudiante, en la cual se puedan dar espacios para incluir preguntas que el entrevistador considere necesarias.

4. El entrevistador debe ser el mismo docente que tiene a cargo el estudiante ciego o con baja visión, este puede pedir ayuda a otros especialistas que considere necesario.

\section{Educación inclusiva}

La educación inclusiva busca satisfacer las necesidades de todos los estudiantes en un aula de clases y no sólo de aquellos que poseen necesidades educativas especiales, es decir, su fin último como lo señala Díaz y Sánchez (s.f) es "conseguir que todos los estudiantes, independientemente de sus circunstancias, tales como cultura, entorno, situación familiar y/o conocimientos previos dispongan de las mismas oportunidades para el aprendizaje" (p. 2). Se recomienda:

1. Concientización a la comunidad educativa sobre la atención a la diversidad y posteriormente sobre las necesidades educativas especiales de la población con discapacidad visual.

2. Capacitación por parte de profesionales en el uso de diversas estrategias, recursos y actividades para incluir a los estudiantes con discapacidad visual.

3. Trabajo en grupo por parte de especialistas para crear las estrategias de aprendizaje de acuerdo a las necesidades de los estudiantes ciegos o de baja visión.

4. Participación activa de los padres de familia y de la comunidad estudiantil en la atención a estos estudiantes.

5. Coordinación con otros docentes que han trabajado en sus aulas con estudiantes ciegos o de baja visión para que aporten su experiencia y en conjunto mejorar las estrategias de aprendizaje a utilizar. 


\section{Mediación Pedagógica}

Los docentes ya no son transmisores de conocimiento, sino mediadores entre éste y el estudiante, buscan que los discentes construyan y se apropien del conocimiento mediante diferentes estrategias en las cuales ellos son guías. Estos guías deben crear en el aula métodos, técnicas y estrategias que permitan respetar la diversidad de sus estudiantes y trabajar en relación a lo que estos necesitan, no solo en la parte de aprendizaje de conocimientos sino también en la convivencia con el grupo de estudiantes. Se recomienda:

1. Diseñar estrategias metodológicas donde se propicie y estimule el trabajo colaborativo de todos los estudiantes para lograr objetivos en común, ya que como lo señala Pujolás (2006)

...si se les orienta y prepara activamente, los propios compañeros son los primeros "aliados" del estudiante con discapacidad, los que mejor acaban conociéndole, y los que más ideas aportan en beneficio de la participación de su amigo con discapacidad en las actividades comunes (p. 15).

2. Diseñar actividades de clase y de evaluación donde se incluyan tanto a los estudiantes ciegos o con baja visión como a los demás.

3. Evaluar si los recursos o actividades planteadas en las estrategias metodológicas logran el objetivo propuesto.

4. Dejar que el estudiante ciego o de baja visión trabaje sólo.

5. Evaluar continuamente el desempeño de todos los estudiantes, tanto de manera individual como grupal.

\section{Diseño universal}

El diseño universal considera las necesidades de toda la población, no se limita al uso de materiales, objetivos, estrategias y evaluación específica, sino que busca dar flexibilidad a estos aspectos, tomando como base las necesidades de la población involucrada para crear estrategias que sean inclusivas y además dirigidas a atender la diversidad, sin embargo, pueden surgir estrategias que funcionen para unos estudiantes y para otros no, por lo que esta flexibilidad se convierte en el aliado más importante del diseño universal. Se recomienda:

1. A partir de la valoración de las capacidades intelectuales, habilidades, condiciones familiares, socioeconómicas y culturales brindar al estudiante diversas alternativas que le permitan acceder al aprendizaje.

2. Crear contextos de aprendizaje flexibles desde el inicio, donde todos los estudiantes puedan personalizar su aprendizaje, es decir, que estos puedan escoger entre múltiples opciones y utilicen aquella que mejor responda a sus capacidades y preferencias.

3. Implementar recursos tecnológicos que faciliten el trabajo en clase tanto del estudiante con discapacidad visual como el que no posee la discapacidad, además que no generen mucho gasto económico y con los cuales el estudiante ciego o con baja visión se sienta a gusto.

\section{Conclusiones}

Hay que concientizar a la comunidad educativa sobre la atención a la diversidad y posteriormente sobre las NEE de la población con discapacidad como se cita en el segundo lineamiento, ya que es un deber de las instituciones públicas proyectar en la sociedad aspectos como el mencionado.

Se debe implementar una metodología participativa y colaborativa que integre a los estudiantes con NEE en el trabajo de aula con el resto de sus compañeros, de esta manera se comenzará a realizar acciones que demuestren la aplicación de una educación inclusiva en los salones de clase de las instituciones educativas, tanto en primaria, secundaria como a nivel universitario.

Es necesario antes de atender a un estudiante con NEE determinar las capacidades intelectuales, habilidades, condiciones familiares, socioeconómicas y culturales del alumno como 
se cita en el primer lineamiento. De esta manera, el docente podrá establecer las condiciones que requiere para brindar una educación de calidad al estudiante.

Se deben diseñar actividades de clase y de evaluación continua donde se incluyan tanto a los estudiantes ciegos o con baja visión como a los demás compañeros y evaluar si los recursos o actividades planteadas logran el objetivo propuesto como se indica en el tercer lineamiento, ya que de esta manera se podrán tomar decisiones para mejorar o continuar con los procesos que se realice, pero todo a partir de la información que se recolecte.

A partir del diagnóstico se debe seleccionar los recursos didácticos que faciliten el trabajo en clase del estudiante con NEE, además que no generen mucho gasto económico y con los cuales el estudiante ciego se sienta a gusto como se cita en el cuarto lineamiento.

A pesar de los esfuerzos que han hecho los organismos internacionales en materia de normativa sobre los derechos e igualdad de las personas con algún tipo de discapacidad, aún persiste en muchos países del mundo la discriminación hacia este tipo de población tanto en el área de la educación como en el de producción de bienes y servicios.

A pesar de los grandes esfuerzos que ha hecho Costa Rica a través de su historia en la atención de estudiantes con NEE tanto en su legislación como la Ley 7600 y la creación instituciones especializadas en el área como el CENAREC, el Instituto Hellen Keller, la Asesoría en Educación Especial del MEP, la Escuela de Educación Especial Fernando Centeno Güell, entre otros, todavía se requiere de muchos recursos económicos para infraestructura y capacitación a los docentes en ejercicio para que puedan atender las diferencias individuales de este tipo de población.

\section{Recomendaciones}

Es importante incorporar en los Trabajos Finales de Graduación de los estudiantes de Licenciatura en las carreras de educación de la UNED proyectos y tesis que tengan que ver con la atención de estudiantes NEE con algún tipo de discapacidad física, visual, motora, entre otras, de manera que se genere literatura y propuestas metodológicas para brindar una adecuada instrucción a los docentes costarricenses.

Es necesario coordinar con las Direcciones Regionales de Educación para establecer capacitaciones a los docentes en ejercicio sobre la atención de estudiantes con NEE, las cuales se pueden realizar en conjunto con las universidades públicas del país.

Es importante que la UNED alfabetice a su personal en la temática sobre la diversidad no solo en aspectos propios de la entrega de la docencia, sino que también administrativos que tengan que ver con la atención de este tipo de estudiantes.

En el diseño de estrategias metodológicas inclusivas que involucren el uso de recursos didácticos, es necesario tomar en cuenta lineamientos que tengan que ver con el diseño universal y la educación inclusiva que considere todas las necesidades de la población estudiantil con NEE o sin ellas.

\section{Referencias Bibliográficas}

Barrantes, K. Rodríguez, A y Rodríguez, S. (2006). El aprendizaje de la Geometría en el nivel de octavo año de la Enseñanza General Básica para estudiantes declarados ciegos legales: Estudio de caso en el Colegio Técnico Profesional de Parrita. Tesis de grado: Heredia, UNA.

Blanco, R. (2009). Experiencias educativas de segunda oportunidad. Lecciones desde la práctica innovadora en América Latina. Recuperado de http://unesdoc. unesco.org/images/0018/001864/186472s.pdf

Cátedra Iberoamericana. (2015). Historia. Recuperado de http://fci.uib.es/Servicios/libros/articulos/di_nasso/ Historia.cid220290

CENAREC. (2012). La educación especial en Costa Rica: antecedentes, evolución, nuevas tendencias y desafíos. Costa Rica: Procesos Litográficos de Centroamérica, S.A.

Costa Rica. (2014). XX Informe del Estado de la Nación. Recuperado de http://www.estadonacion.or.cr/20/assets/cap-2-estado-nacion-20-2014-baja.pdf 
Costa Rica. Leyes, decretos, (2004). Ley 7600 sobre igualdad de oportunidades para las personas con discapacidad. San José: EDITORAMA

Díaz, E y Sánchez, S. (s.f). La educación inclusiva desde el curriculum: el diseño universal del aprendizaje. Recuperado de https://www.researchgate. net/publication/261833343_LA_EDUCACION_ INCLUSIVA_DESDE_EL_CURRICULUM_el_ Diseno_Universal_para_el_Aprendizaje

Fernández, A. (2003). Educación Inclusiva: "Enseñar y Aprender entre la Diversidad". Recuperado de http:// www.inclusioneducativa.org/content/documents/ Generalidades.pdf,

Fundación Centros de Aprendizaje. (2010). Orientaciones pedagógicas para la inclusión de estudiantes en situación de discapacidad. Descargado de http://www. included.eu/sites/default/files/documents/colombia. orientacionesinclusion.pdf

Gutiérrez, F y Prieto, D. (2002). Mediación pedagógica. Universidad de San Carlos de Guatemala: Guatemala. EDUSAC.

Hernández, R., Fernández C. y Baptistas, M. (2010). Metodología de la Investigación. Mac Graw Hill: México D. F.

Hirmas, C. (2008). Educación y Diversidad Cultural: Lecciones desde la práctica innovadora en América Latina. Recuperado de http://unesdoc.unesco.org/ images/0016/001626/162699s.pdf

Marín, M. (2011). Alumnos con Necesidades Educativas Especiales. Costa Rica: EUNED.
Meléndez, L. (2010). La educación especial en Costa Rica. Costa Rica: EUNED.

MEP. (2005). Normas y Procedimientos para el Manejo Técnico-Administrativo de los Servicios Educativos para Estudiantes con Discapacidad Visual. Costa Rica: Litografía e Imprenta LIL, S.A.

MEP. (2008). El centro educativo de calidad como eje de la educación costarricense. Recuperado de http:// www.mep.go.cr/sites/default/files/page/adjuntos/ centro-educativo-calidad-como-eje-educacioncostarricense.pdf

PRELAC. (2002). Proyecto Regional de Educación para América Latina y el Caribe. Recuperado de http://portal.unesco.org/geography/es/ev.php-

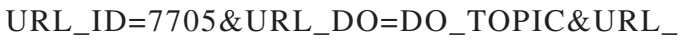
SECTION=201.html

Pujolás, P. (2006). Aulas inclusivas y aprendizaje cooperativo. Descargado de http://www.unizar.es/cce/atencion_diversidad/ap\%20cooper.pdf

Rojas, E y Sequeira, R. (2012). Recursos didácticos en Matemáticas. Costa Rica: EUNED (versión preliminar).

UN. (2015). Declaración Universal de los Derechos Humanos. Recuperado de http://www.un.org/es/ documents/udhr/

Vásquez, P. (2012). Sistematización Taller Estrategias Inclusivas para el Desarrollo de las Matemáticas en Secundaria. Cátedra Educación Especial, UNED. 
